\title{
Humanismo educativo en la sociedad del conocimiento
}

\section{Educational Humanism in the society of knowledge}

Carlos Luis Chanto Espinoza

Universidad Nacional, Sede Regional Chorotega

Liberia, Costa Rica

carlos.chanto.espinoza@una.cr

Marlene Durán López

\begin{abstract}
Resumen
El paradigma humanista en la educación refleja el interés del ser humano por superar vacíos que la educación tradicional u otras ideologías han dejado en el ser. Por ello, el reconocimiento del potencial y las cualidades individuales representan una necesidad que debe ser abarcada y acatada por el sector educativo, con miras a brindar un mejor apoyo a la formación y consolidación pedagógica en nuestras sociedades.

La contextualización de nuestra realidad educativa propone cambios de pensamiento y metodología ante las necesidades de la población estudiantil. Ante este panorama se dejan ver y escuchar movimientos encaminados a preparar y enfrentar al estudiante al contexto, lo cual representa un reto en la nueva sociedad de conocimiento, que requiere acciones concretas desde las aulas para la formación humana e integral.
\end{abstract}

Palabras claves: sociedad de conocimiento, TIC, educación humanista, sociedad de información. 


\begin{abstract}
The humanist paradigm in education reflects the interest of human beings to overcome the gaps that traditional education or other ideologies have left in them. That is why, the recognition of the potential and of individual qualities represents a need that has to be fulfilled and observed by the public sector in order to offer better support to the development and pedagogic consolidation of our society.

The contextualization of our educational reality proposes changes of thought and methodology according to the needs of the students. In view of this scenario, it is easy to see and to hear about movements aimed at preparing the students and making them face the context in which they get involved, which represents a challenge in the new society of knowledge that requires concrete actions in the classroom for the benefit of human and integral growth.
\end{abstract}

Keywords: society of knowledge, Information technology, humanist educator, technological society

\title{
Introducción
}

A través de la historia, las colectividades han procurado, a su manera, ser sociedades del conocimiento. El acceso y empoderamiento de este se mantuvo acaparado por un grupo con ciertas características diferenciadas, que presentaron ventajas y dominio del conocimiento, y le concedieron ese privilegio a un círculo muy cerrado de sabios. Esta propagación de conocimiento fue trasmitida de generación en generación, en sus inicios de forma verbal, luego por los manuscritos y con pasar del tiempo por medio de los libros, gracias a la revolución que trajo consigo la imprenta, hasta llegar a lo que hoy conocemos como tecnologías de información y comunicación (TIC).

La evolución histórica hacia esta apertura ha estado sustentada en la educación para todos y apoyada por las luchas sociales en contra de las desigualdades y exclusiones vividas a través de los tiempos. Por ello, en una sociedad de información y conocimiento, la educación humanista toma auge en miras de propiciar y abrir nuevas perspectivas a un conocimiento público, auténtico e igualitario que se fortalezca de las capacidades y pueda respetar las diversidades.

Por ende, es menester propiciar la apertura al conocimiento, mediante acciones que consoliden una educación humanista, que involucre los diferentes actores, 
de manera que puedan utilizar eficazmente las herramientas que nos proporciona la sociedad de la información, que tome en cuenta la individualidad y que a la vez fortalezca el aporte de equipo que puede brindar la diversidad con miras de consolidar la sociedad de conocimiento.

\section{Educación Humanista}

Para comprender el Humanismo, es necesario primero conocer su significado de acuerdo con el contexto histórico, social, cultural, espiritual, además de otros aspectos que a través de las épocas han dado diferentes connotaciones. Sin embargo, cada aporte ha contribuido a entenderlo como lo percibimos hoy.

El concepto de humanismo, con su implicación en aspectos pedagógicos, ha dado paso al concepto de educación humanista, el cual resulta controversial, pues parte del hecho de que toda educación debe ser humanista y que, por lo tanto, propicia las condiciones de desarrollo intelectual e integral del estudiante.

Según García (s.f.) la educación humanista se define como de tipo indirecto, pues en ella el docente permite que los alumnos aprendan, mientras impulsa y promueve todas las exploraciones, experiencias y proyectos que estos preferentemente inicien o decidan emprender, a fin de conseguir aprendizajes vivenciales con sentido.

A su vez, Patiño (2007) define la educación humanista más allá del desarrollo intelectual cultural y crítico, y retoma la dimensión que tiene que ver con la necesidad espiritual de acercamiento a la realidad trascendente, a Dios.

Estas definiciones comparten el pensamiento sobre un aprendizaje más humanizado, para lograr mejores resultados al conocer experiencias o situaciones que puedan afectar de una u otra forma el rendimiento del estudiante, pues como seres integrales nos afectan y desequilibran circunstancias, que de ser tratadas o canalizadas, logran que el estudiante un ser reflexivo y propositivo en la búsqueda de soluciones.

La educación humanista representa en los tiempos actuales una vislumbre hacia un paradigma que visualiza las necesidades individuales y pueda responder acertadamente a cada estudiante, de acuerdo con sus divergencias $y$, a su vez, fomente las iniciativas y el potencial creativo de cada alumno en una sociedad de competencias y conocimiento. 


\section{Perfil del educador Humanista}

La participación del profesor, como un ente canalizador, desempeña rasgos significativos que deben ser aplicados con miras de su rol formador, generador y no simplemente trasmisor. Entender la nueva posición del estudiantado en la nueva sociedad implica cambios significativos de actitud en todos los actores involucrados con nuevas experiencias creativas e innovadoras que fomenten su desarrollo en todas sus áreas.

Ser un docente humanista requiere compromiso, dedicación y tiempo, la experiencia en el ámbito educativo ha reflejado la necesidad que tienen los estudiantes de encontrar personas que se interesen en ellos y presten atención a sus inquietudes, propuestas u opiniones. A continuación, se presentan características sobresalientes del perfil de este tipo de docente:

- Escucha a las personas y es humilde para reconocer que los estudiantes tienen mucho para enseñar.

- Se interesa no solo en que el estudiante adquiera conocimientos, sino también en su desarrollo integral.

- Respeta la libertad de opinión, sin tendencias a reprimirla.

- Se apropia de las tendencias educativas dirigidas a mejorar el aprendizaje.

- Es cooperativo y promueve el aprendizaje colaborativo.

- Busca alternativas de solución ante dificultades que se puedan presentar en el proceso de enseñanza o en situaciones particulares del estudiante, actúa con respeto y sensibilidad.

- Posee una actitud receptiva ante las críticas o recomendaciones.

- Genera aprendizaje en lugar de simplemente transmitir conocimiento.

- No es arrogante.

Este docente promueve la expresión, comunicación, investigación y la formación autodidacta para fortalecer al individuo, de modo que logre apropiarse de conocimientos base, los cuales permitan formar un criterio y realizar la acertada toma de decisiones ante las olas de pensamiento de la sociedad de información en la que estamos inmersos. Por ende, urge la concienciación de no ser partidarios de un ser acumulador de datos, simplemente.

La contextualización a nuestra realidad educativa propone cambios de pensamiento y metodología para muchos docentes que anteponen su dominio a las necesidades de la población estudiantil, lo cual nos lleva a una reflexión sobre lo que estamos haciendo y 
lo que podemos cambiar y llegar hacer, con el fin de llevar iniciativas a las aulas y lograr el aprendizaje deseado y el desarrollo de las facultades, no solo de los estudiantes, sino de todos los actores involucrados en este proceso. Esto nos llevará por un camino donde hay que desaprender para volver a aprender mejores prácticas, que fomenten el valor del ser humano como un ente social, individual e integral, con destrezas necesarias para su inmersión en la sociedad del conocimiento y de la información.

\section{Humanismo en la sociedad del conocimiento y de la información}

El nuevo siglo ha traído fenómenos complejos que han originado diversas conceptualizaciones y adjetivaciones, los cuales procuran contribuir a la comprensión de las nuevas dinámicas socioculturales que vive la humanidad. Las TIC dieron paso a la sociedad de información y al mismo tiempo a la sociedad del conocimiento, cuya diferencia la ilustra Toledo de la siguiente manera:

La diferencia entre la Sociedad de la Información y la Sociedad del Conocimiento, que propugna la declaración de Bolonia es que entre uno y otro proceso está el aprendizaje, en el marco de una formación humanista que reivindique para el estudiante la conciencia de su papel participativo en la transformación social y productiva. No se trata, por lo tanto, de manejar información, sino de convertirla a través del aprendizaje en conocimiento, única manera que le permitirá tomar decisiones y desarrollar acciones de futuro, siempre en el marco de la pertinencia, la calidad y la internalización que proponen tanto los documentos de la conferencia Mundial como la Declaración de Bolonia. (2010)

De la cita anterior, podemos destacar que no toda información es conocimiento. Esto es un asunto complejo que va mucho más allá de los datos que se recolectan cuantitativamente en un archivo o incluso en una base de datos. La sociedad originada a partir de esta detonación de los medios de comunicación, no es del conocimiento, sino más bien una sociedad de la información, de la recolección de datos que producen acaparamiento de riqueza material e incluso del poder.

Actualmente, vivimos en una sociedad de acopio de información que tiene diferentes niveles de transparencia y de relación con las situaciones humanas, las cuales no se diferencian y forman una especie de estandarización de saberes que coloca en interrogantes la conveniencia y los impedimentos éticos de estos datos recolectados.

Formar seres humanos con perspectivas integrales en una sociedad que se caracteriza por el fenómeno de la sociedad de la información es todo un reto, el cual va excavando, en gran medida, la capacidad de reflexionar, analizar, distinguir, cuestionar, procesar y tomar 
una actitud sobre los datos multiplicados con rapidez en la Internet. Hallamos diseños teóricos que requieren desarrollar y explorar los desafíos dibujados por la sociedad del conocimiento a la alineación humanista, reflexiones sobre la ética profesional en estudiantes y profesores, que dejan ver el cambio en métodos de evaluación.

Debe existir un desafío de vínculo social entre el sector educación con las organizaciones de la sociedad civil, para suscitar el desarrollo humano y el comunitario en un tiempo evidente por el individualismo, el esbozo de un caso de formación humanista y sus desafíos ante el transcurso de globalización o trabajos que recapacitan sobre la gestión del conocimiento desde una apariencia humanista en esta sociedad de la información y del conocimiento.

El informe denominado "Reinventar las telecomunicaciones", publicado por la Unión Internacional de Telecomunicaciones, sobre el Desarrollo de las Telecomunicaciones en el 2002, señala la forma como se pueden utilizar estas nuevas tecnologías para alcanzar los objetivos planteados en la Declaración del Milenio y algunas utilidades de las tecnologías agrupadas, según un objetivo. De dicho informe, a continuación se extraen los siguientes puntos transcendentales:

\section{Reducir la pobreza:}

- Aumentar el acceso a la información comercial y reducir los costes de las transacciones a favor de las economías menos desarrolladas.

- Fomentar la capacidad de los países en desarrollo para participar en la economía mundial y aprovechar sus ventajas comparativas.

- Acrecentar la eficiencia, la capacidad de competir y el acceso a los mercados de las empresas de los países en desarrollo.

\section{Educación e inclusión:}

- Aumentar el número de profesores capacitados, mejorando su capacitación gracias a las TIC, la formación a distancia (e-learning) y redes de conocimiento que vinculen a unos profesores con otros.

- Ampliar la disponibilidad de materiales y recursos docentes de calidad mediante las TIC.

- Impartir programas de educación y alfabetismo, especialmente aquellos destinados a grupos con riesgo de exclusión, mediante las tecnologías idóneas. 
Estas propuestas constituyen un reto, en el cual es clave entender que todos somos participes en lograr la sociedad igualitaria, que trasciende las dimensiones sociales, éticas y políticas en busca de un desarrollo humano sostenible.

\section{Hacia una educación humanista}

La educación, desde el nivel más básico hasta el grado académico más alto (doctorado o posdoctorados), forma cada generación para insertarse, retribuir y aportar para la construcción de una sociedad más justa e imparcial. Se debe luchar por una sociedad más equitativa y solidaria con el auxilio del sistema educativo, lo cual implica iniciativas políticas que den esperanzas y estímulo a ese arduo compromiso. Actualmente, con la ayuda de las tecnologías de información como herramienta de apoyo para los estudiantes, se trabaja para estimular la ambición por aprender y satisfacer las necesidades de conocimiento.

La educación en nuestros días presenta una preocupación para nuestra sociedad, ante la tendencia de los jóvenes por aprender apresuradamente, con el fin de incorporarse con mayor rapidez a la fuerza de trabajo de la sociedad. La actividad educativa y la inmersión en un mundo tan acelerado, dificultan la visión de calidad, donde instruir se ha convertido en un asunto mecánico dirigido a obtener productos explícitos.

Desde esta perspectiva, se considera el trabajo de formar a los educandos como una responsabilidad de inigualable compromiso, ante la huella que como docente se dejará en el estudiante y la de este en la sociedad; por ende, urge que el estudiante se desarrolle plenamente como persona, a sabiendas de que el objetivo final de la educación no es la perfección; por el contrario, es la preparación para la vida. Debe considerarse la existencia de ciertas habilidades en la naturaleza humana, frecuentes en todos, las cuales componen el caudal de las energías principales del hombre, sin importar su clase social y vocación.

\section{La escuela hoy}

Un centro de estudios (escuela, colegio, universidad), en sentido extenso, representa un lugar donde es viable que el ser humano se desarrolle en su humanidad. La calidad del centro radica esencialmente en la comunicación desde la reciprocidad enseñanza-aprendizaje. El crecimiento de las nuevas tecnologías de la información y la comunicación ha fundado nuevos contextos para la 
aparición de sociedades del conocimiento, las cuales buscan la edificación a nivel mundial de sociedades que sean nacimientos de progreso.

Para conseguirlo, se deben tener presentes dos desafíos trazados por la revolución de la información, los cuales recubren una importancia específica: el acceso a la información para todos y el futuro de la libertad de expresión. Las tecnologías de la información y las comunicaciones (TIC) se han transformado en una herramienta imprescindible para la disputa contra la pobreza, usualmente una obligación para el desarrollo.

Con ellas las naciones en desarrollo tienen una oportunidad sin antecedentes de propiciar efectivamente objetivos de desarrollo de principal necesidad, como la disminución de la pobreza y suministro de servicios básicos de salud y educación, entre otros.

\section{Las sociedades del conocimiento, fuentes de desarrollo}

Un componente central de las sociedades del conocimiento es la cabida para identificar, transformar y difundir, así como aplicar conocimientos necesarios para el desarrollo humano. Las sociedades se basan en un enfoque de la sociedad que atenúa la independencia y comprende elementos de diversidad, composición, apoyo y colaboración.

La posición basada en el "desarrollo humano" y la "autonomía" es un componente central de la generalidad de sociedades del conocimiento. Esto debería aprobar una mejor puesta en práctica de los derechos universales y las libertades fundamentales, los cuales perfeccionen, al mismo tiempo, la minimización de la pobreza; y el aumento de las políticas de desarrollo.

El auge de las sociedades del conocimiento exige que se sujeten nuevas relaciones entre el conocimiento y el desarrollo, porque este es un instrumento para indemnizar las necesidades económicas, como un elemento colmado de progreso. Replantear la dimensión del aprendizaje en las nacientes sociedades del conocimiento, no parece tarea fácil; debemos quizás, enmarcar la cultura de la innovación, en todos los ámbitos de la dinámica humana, con la edificación y disponibilidad de los conocimientos, como características más importantes en el desarrollo de las sociedades del aprendizaje.

Por ende, libertad de palabra y autonomía son, entre otros, derechos fundamentales, donde es obligatorio realzar liberación de expresión. Valores como la libertad de expresión y la libertad de investigación científica, acompañadas de un espíritu crítico y ético permiten construir legítimas sociedades del conocimiento. 


\section{¿Hacia una sociedad mundial de la información?}

En el plano universal, los elementos de desigualdad ante los acaecimientos tecnológicos se conciertan al crear una genuina brecha digital universal, la cual pone en el lienzo de juicio la generalidad del desarrollo de las nuevas tecnologías. Si el sistema directo particular de la Internet intentaba prometernos una eliminación temporal de los instrumentos de las distancias y el alejamiento. La presencia de la brecha digital nos recuerda que se sigue viviendo una geografía del ciberespacio.

En efecto, las desigualdades en materia de dotación industrial inducen a desigualdades de desarrollo en las infraestructuras, las cuales son el motor de la difusión de las nuevas tecnologías. Por lo tanto, se puede decir que existe efectivamente una correlación entre las desigualdades en materia de desarrollo industrial y las disparidades en el acceso a la información.

Las TIC crean la base tecnológica para la Sociedad de la Información. De forma espontánea, la "brecha digital" es una expresión utilizada para opinar que entre países y entre desiguales grupos de personas dentro de un país haya una extensa diferencia entre quienes tienen acceso a las TIC y quienes no lo poseen. Son, cada vez más, una columna para la edificación de las sociedades y economías presentes. Una de las consecuencias de la brecha digital en las poblaciones que no tienen acceso a la información es la pérdida de oportunidades para resolver algunas carencias primordiales de desarrollo.

La brecha digital es un espejo de otras privaciones, como sociales y económicas. No obstante, todos los países, incluso los denominados países más pobres, han desarrollado su acceso y utilización de las TIC; asimismo, países desarrollados se han adelantado de forma exponencial, de modo que las diferencias han continuado aumentando. Dentro de cada país, está ocurriendo algo semejante, por lo cual la brecha interna también progresa.

Sin embargo, cabe recalcar que el sector educativo ha apostado al uso de la tecnología en las aulas y, por ende, hoy día vemos pasos encaminados a que los estudiantes puedan tener acceso a las diversas tecnologías desde sus centros de enseñanza. Además de este avance, se requiere una educación humanista que logre aprovechar los recursos de que dispone la institución para procurar e incentivar en el estudiante la búsqueda de conocimiento y el uso ético de estas herramientas en pro de su bienestar y el de la sociedad. 


\section{Consideraciones finales}

Según la Organización de las Naciones Unidas para la Educación, la Ciencia y la Cultura (UNESCO, 2005) en su Informe Mundial de la UNESCO: Hacia las sociedades del conocimiento, recalca la necesidad de "actuar para que los conocimientos se articulen con las nuevas formas de elaboración, adquisición y difusión del saber valorizadas por el modelo de la economía del conocimiento" en una sociedad que se nutre de sus diversidades y capacidades.

Producto de esta declaración y como se menciono anteriormente, el sector educativo ha sido y es el ende desde el cual se debe propiciar la apertura al conocimiento autentico que tome en consideración al ser humano en su dimensión integral y logre favorecer y fortalecer sus facultades y diversidades con miras a una educación de calidad para la vida.

Para lograr este cometido, cada persona debe ser diferenciada y abstraer sus particulares necesidades, lo cual representa una complejidad y un reto a abordar desde el sector educativo; si bien es cierto, los planteamientos están en la teoría, estos difieren en la práctica, donde muchas veces quedan vacíos sobre cómo proceder ante circunstancias presentadas por la persona, el grupo o el modelo pedagógico por seguir.

Al ser el estudiante la razón primordial del proceso educativo, surge la pregunta de si realmente nuestra educación contiene el ingrediente humano y si como docentes somos eficientes en la generación de aprendizaje y no meramente en la enseñanza de teorías y conocimientos, en una sociedad tan acelerada por las TIC.

Desde este argumento, la formación del educador en TIC posee un doble significado: la necesaria apropiación de un conjunto de capacidades en estos temas como instrumentos para cualquier profesional en un mundo altamente tecnológico $\mathrm{y}$, en segundo lugar, la apropiación de las TIC como una eficaz herramienta para convertir y mejorar la enseñanza y el aprendizaje, sin dejar de lado la parte humana.

Necesitamos un cambio metodológico obligatorio que permita integrar las TIC en el currículo. Las TIC son parte del mundo de nuestros estudiantes (Facebook, YouTube, Google, entre otras). Ellos ya saben usarlas con muchas otras finalidades relacionadas con el ocio. Con todo esto, la idea no solo es consultar la información, a modo de libros de texto, sino procurar crear nuevo conocimiento, nuevos materiales y herramientas, a partir de las posibilidades que nos brindan. 
Los cambios medulares en el paradigma educativo deben surgir como respuesta a las nuevas súplicas sociales, lo cual implica obligatoriamente que las nuevas tecnologías sean competentes para atender uno de los principales objetivos de la educación: el proceso emocional de los educandos y su formación.

La búsqueda de nuevas fases sociales y educativas difícilmente será posible si las TIC continúan siendo herramientas técnicas utilizadas únicamente para la adquisición y transferencia de conocimientos, pero inhábiles de informar y contribuir con los objetivos sociales que apremian la educación y la sociedad.

La incorporación de las TIC en la educación, como ya se ha definido, no garantiza por sí sola la introducción y el equilibrio social. El reto se fundamenta en ser capaces de continuar transfiriendo los valores perenemente a través de los diferentes medios y que a la vez promuevan el aprendizaje para que las nuevas generaciones logren desenvolverse dentro de la cultura digital de una manera responsable.

Desde el ambiente académico, se debe beneficiar la revolución tecnológica para que se proporcione la creación de conocimientos, donde se consoliden sociedades intelectuales que resguarden la educación integral dentro de las políticas de desarrollo, en procura que la educación sea un elemento para distribuir e incrementar el bienestar de la sociedad y así alcanzar el desarrollo en todas sus formas.

\section{Referencias}

Aguilera, J. Galvez, L. (2004). La gestión educativa desde una perspectiva humanista. Buenos Aires: Ril.

CEPAL (Comisión Económica para América Latina). (2005). Políticas públicas para el desarrollo de sociedades de información en América Latina y el Caribe. Recuperado de: http://www.eclac.cl/publicaciones/xml/5/21575/Politicas\%20Publicas.esp.pdf

Cumbre Mundial de la Sociedad de la Información. (2003). Principios de Ginebra. Recuperado de: http://www.itu.int/wsis/docs/geneva/official/dop-es.html

García, J. (s.f.). ¿Qué es el paradigma humanista en la educación? Recuperado de: http://www.riial.org/espacios/educom/educom_tall1ph.pdf

Gil, M. (2012, Julio - diciembre). Primer Congreso Nacional de Información para el Desarrollo: "Costa Rica hacia la sociedad de la información de todos para todos": Informe final mesas de trabajo. E-Ciencias de la Información, 2(2) Recuperado de: http://revistaebci.ucr.ac.cr/volumenes/2/2-2/2-2-1nt/2-2-1nt.html 
Ministerio de Ciencia y Tecnología. (2003). La Sociedad de la Información en el Siglo XXI: un requisito para el desarrollo. Buenas prácticas y lecciones aprendidas. España. Recuperado de: http:/www.itu.int/wsis/stocktaking/docs/activities/1103547250/ sociedad-informacion-sigloxxi-es.pdf

Patiño, H. (2007). La Universidad de Inspiración Cristiana en Tiempos de Poscristiandad. Recuperado de: http://books.google.co.cr/books?id=lqtSJlAA-xcC\&pg=PA147\& $\mathrm{dq}=$ educacion + humanista\&hl=es-419\&sa=X\&ei=KJ-eUbnkC5L68QTcmYCoD A\&ved $=0$ CFsQ6AEwCQ\#v=onepage \&q=educacion $\% 20$ humanista\& $\mathrm{f}=$ false

Sortilegios educativos (2009). Propuesta educación alternativa. Recuperado de: http:// sortilegioseducativos.com/

Toledo, M. (2010). El resumen automático y la evaluación de traducciones en el contexto de la traducción especializada. Alemania: Peter Lang.

UNESCO (Organización de las Naciones Unidas para la Educación, la Ciencia y la Cultura (2005). Informe Mundial de la UNESCO: Hacia las sociedades del conocimiento. Ediciones UNESCO. Recuperado de: http://unesdoc.unesco.org/ images/0014/001419/141908s.pdf

Unión Internacional de las Telecomunicaciones. (2003). Informe sobre el desarrollo mundial de las telecomunicaciones: Indicadores de acceso para la sociedad de la información. Recuperado de: http:/www.itu.int/ITU-D/ict/publications/wtdr_03/ material/WTDR03Sum_s.pdf 